\title{
Concurrent stone stabilization improves ultrasonic and pneumatic efficacy during cystolithopaxy: an in vitro analysis
}

\author{
Shubha De ${ }^{1}$, Carl Sarkissian ${ }^{1}$, Giovanni Marchinni', Manoj Monga ${ }^{1}$ \\ ${ }^{1}$ Glickman Urologic and Kidney Institute, Cleveland Clinic Foundation, Cleveland, Ohio, USA
}

\section{ABSTRACT}

Objective: To identify whether stabilization of larger bladder stones would improve the efficacy of combination (ultrasonic/pneumatic) lithotripsy in a phantom bladder stone model for percutaneous cystolithopaxy.

Materials and Methods: Using $1 \mathrm{~cm}$ phantom Bego stones, a spherical model bladder was used to simulate percutaneous bladder access. A UroNet (US Endoscopy, USA) was placed alongside a Swiss Lithoclast probe through the working channel of a Storz $26 \mathrm{Fr}$ rigid nephroscope. Using a 30Fr working sheath, the stone was captured, and fragmented for 60seconds.

Resulting fragments and irrigation were filtered through a $1 \mathrm{~mm}$ strainer, and recorded. Five trials were performed with and without the UN. Durability was then assessed by measuring net defects, and residual grasp strength of each instrument. Descriptive statistics (mean, standard deviations) were used to summarize the data, and Student's t-tests $($ alpha $<0.05)$ were used to compare trials.

Results: The mean time to stone capture was 12s (8-45s). After fragmentation with UN stabilization, there were significant improvements in the amount of residual stone (22\% dry weight reduction vs $8.1 \%$ without UN, $\mathrm{p}<0.001$ ), number of fragments (17.5 vs 5.0 frag/stone, $p=0.0029)$, and fragment size ( $3.6 \mathrm{~mm}$ vs. $7.05 \mathrm{~mm}, \mathrm{p}=0.035)$. Mesh defects were noted in all nets, ranging from 2-14 mm, though all but one net retained their original grip strength $(36.8 \mathrm{~N})$.

Conclusions: Bladder stone stabilization improved fragmentation when used in conjunction with ultrasonic/pneumatic lithotripsy. However, due to limitations in maneuverability and durability of the UN, other tools need to identified for this indication.
ARTICLE INFO

\section{Key words:}

Urinary Bladder Calculi;

Lithotripsy; Urinary

Int Braz J Urol. 2015; 41: 134-8

Submitted for publication:

March 10, 2014

Accepted after revision:

July 28, 2014

\section{INTRODUCTION}

Bladder stones account for 5\% of urolithiasis in industrialized countries. They are most commonly associated with urinary stasis caused by bladder outlet obstruction (1). Endoscopic management of bladder stones utilizes similar equipment and techniques as kidney stones, however poses several unique challenges.
Endoscopic lithotripsy may be performed by a transurethral or percutaneous suprapubic approach using regular nephroscopy equipment. By creating a $1 \mathrm{~cm}$ skin incision, the percutaneous Seldinger technique for bladder access remains pre-peritoneal and does not require a formal open cystotomy. Using a sheath, fragmentation may be performed using any lithotripter through a nephroscope. The challenges shared by each lithotripter is 
localization and stabilization for fragmentation and retrieval.

The stakes for complete stone clearance are high; retained fragments (especially in B00) are a risk for future stones, acute urinary obstruction, and persistent lower urinary tract symptoms. This technique has been used safely in adults, pediatrics, and in native, augmented, and neo-bladders (2-6). The obstructive process inciting stone formation can also lead to bladder distention, diverticula formation, and chronically infected and inflamed mucosa.

Conduits, neobladders, and augmentation cystoplasties possess multiple folds, debris, mucus, foreign bodies (i.e. extruded clips and sutures). These not only increase the risk of bladder stones up to $40 \%$ in patients at two years (7), but can increase the surgical time due to stone localization, fragmentation, and retrieval. Theoretically the risk of endoscopic bladder stones management in enteric augments/conduits is increased (compared to native bladders) due to the lack of a substantial muscularis layer, and unexpected vascular injuriy during percutaneous access.

In order to reduce stone debris spillage and improve fragmentation, our objective was to study bladder stone stabilization. As no current studies have assessed simultaneous stabilization and lithotripsy through a single scope during cystolithopaxy, we assessed a novel technique using an in vitro bladder stone model, with a new entrapment device. By stabilizing a stone during fragmentation, we hypothesize that we can improve efficiency of ultrasonic and pneumatic lithotripsy.

The entrapment device used in this experiment was the UroNet Retriever ${ }^{\mathrm{TM}}$ (UN), an adaptation of gastroenterology's endoscopic RothNet, constructed to fit flexible and rigid scopes. Though its intended use is to gather coarse fragments after fracture, we sought to study its role in stone stabilization during fragmentation.

\section{MATERIALS AND METHODS}

Phantom Bego stones were created to $1 \mathrm{~cm}$ maximum diameter, and $1 \pm 0.05$ grams. BegoStone Plus $^{\mathrm{TM}}$ plaster (Bego Dental, Bremen, Germany) was used with a 5:1 ratio of Bego power to water, and spherical molds were used for consistent sizing. All stones were dried and weighed prior to usage. A $600 \mathrm{cc}$ round bottom rigid plastic container and urethane cover were used as a bladder model. A central aperture, accommodating a 30Fr sheath was fashioned allowing for rotational and depth manipulation of the nephroscope (modeling the mechanics of a percutaneous cystolithopaxy). A Karl Storz26 Fr rigid nephroscope was used with saline irrigation and Swiss Lithoclast ${ }^{\mathrm{TM}}$ probe (Electro Medical Systems, Nyon, Switzerland) to provide simultaneous ultrasonic and pneumatic fragmentation with continuous suction. The Swiss Lithoclast ultrasound probe used was $11.4 \mathrm{Fr}$, and $403 \mathrm{~mm}$ in length (FR-083), with a 3Fr pneumatic combination probe (EL-220) (Figure-1).

A phantom stone was placed in the bladder and subjected to 60 seconds of fragmentation, with or without UN stabilization (through the nephroscope) using a single operator. The UroNet Flexible Retriever (product no. 913604) produced by US Urology of US Endoscopy (Mentor, OH), has working length of $70 \mathrm{~cm}, 5.4 \mathrm{Fr}$ sheath diameter, and a 3.0x $1.25 \mathrm{~cm}$ nylon net. Video endoscopy for scope manipulation was used to simulate routine surgical practice. Once located with the scope, the UN was advanced through the working channel, and the stone was captured.

Once stabilized by the net, the SL probe was advanced along side the UN through the

Figure 1 - Schematic bladder stone model.

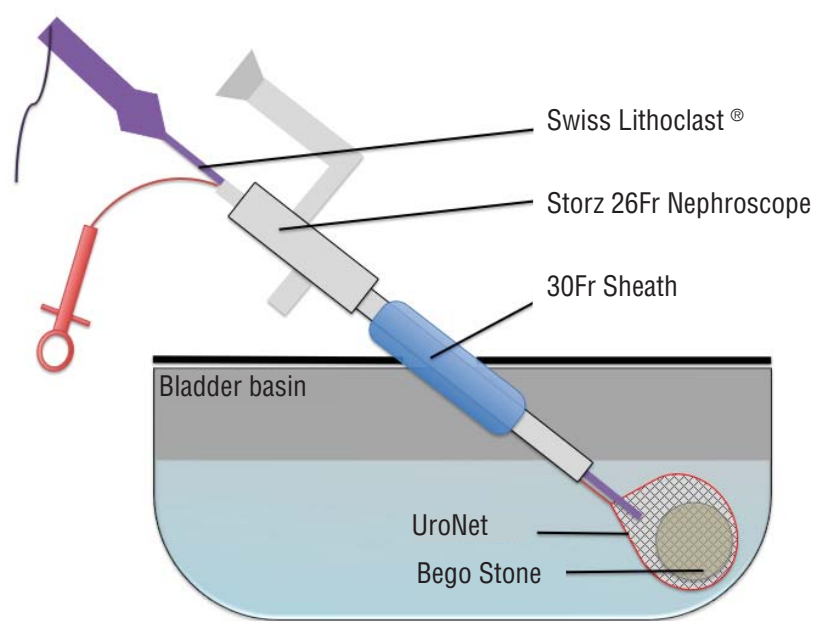


working channel and directed on to the captured stone. Ultrasonic and pneumatic lithotripsy were used simultaneously at maximal power settings for 60 seconds. Stones and irrigation were then sifted through a $1 \mathrm{~mm}$ strainer, and all retained fragments were recorded. Five trials were performed with and without the UN, during fragmentation. The number of fragments, maximum diameter of fragments, and change in dry weight were recorded. Durability was assessed after fragmentation by measuring netting defects and the change in grasp strength of the instrument.

Grasp strength testing involved trapping an $8 \mathrm{~mm}$ marble, and recording the closure force required to fracture the netting around the marble. These forces were then compared to brand new nets, and assessing the variation.

Means and standard deviations were used to summarize the data. Two tailed Student's T-tests were used (alpha <0.05) to compare results with and without UN. SPSS (version 20, Armon NY) was used for all statistical analyses.

\section{RESULT}

Mean time to stone capture, using UN through the nephroscope was 12s (range 8-45s). The average pre-fragmentation dry weight was similar for both trials (1.05 g+UN vs $1.02 \mathrm{~g}-\mathrm{UN})$, and diameters were standardized to $1 \mathrm{~cm}$ using the same mould for all stones.

After 60seconds of SL fragmentation, all UN captured stones were removed by withdrawing the pouch through the sheath. The remaining stones were collected from the strainer, and dried for one week. The change in dry weight when using UN was $22.6 \pm 0.02 \%$, and $8.1 \pm 0.04 \%$ without UN $(\mathrm{p}<0.001)$. Lithotripsy with UN led to greater fragmentation $(17 \pm 5$ vs $5.0 \pm 4.1$ fragments/stone, $p=0.0029$ ) and smaller average fragment size $(3.6 \pm 0.51 \mathrm{mmvs} 7.05 \pm 3.0$ $\mathrm{mm}, \mathrm{p}=0.035$ ). Defects in the meshwork ranged from 2-14 $\mathrm{mm}$, though all nets retained their original strength $(36.8 \mathrm{~N})$, except one which was nonfunctional. This net developed a $14 \mathrm{~mm}$ defect too large to grasp the $8 \mathrm{~mm}$ marble used for testing (Figure-2).
Figure 2 - UroNet Retriever with $4 \mathrm{~mm}$ mesh defect after lithotripsy.

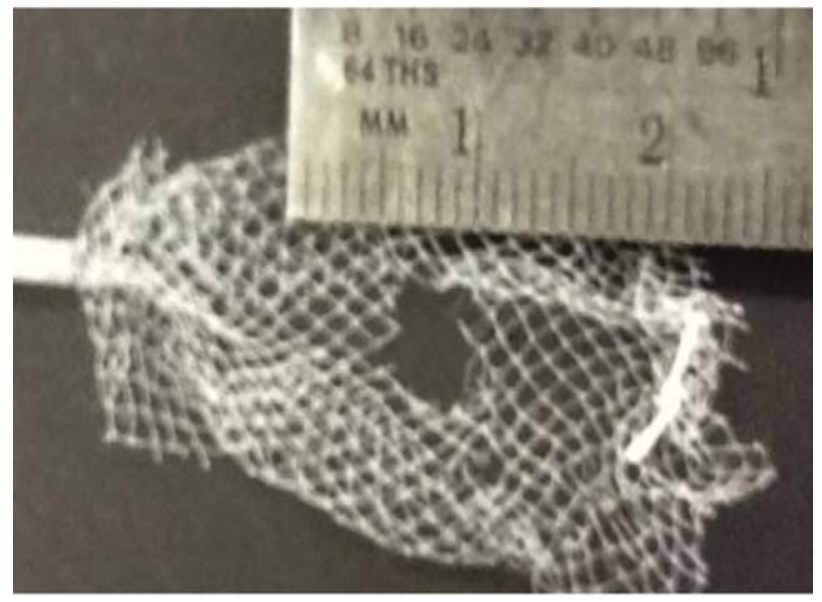

\section{DISCUSSION}

Endoscopic management of bladder stones offers unique challenges, some of which can be addressed by simultaneous fragmentation and stabilization. In conjunction with dual-modality lithotripsy provided by SL, the UN aided in improving fragmentation, and significantly reduced the amount of residual stone after 60s of breakage. This advantage is not limited to a percutaneous procedure, as it could easily be performed transurethrally. However, though improvements in fragmentation efficacy were identified, several issues concerning the UN design, prevents it from being the ideal tool for this indication.

Stone capture was straight forward, though controlling both the SL and UN through the working channel could be challenging. As the net closes around a stone it is tethered at the shaft's insertion, producing a fulcrum. As they exit the scope in parallel, depth becomes the probe's major degree of freedom. With minor adjustments of the nephroscope and rotation of the UN, the captured stone can be rolled into the probe's path. Once aligned, little manipulation is needed as probe suction keeps the netted stone in place. If the option was available to remove the UN sheath, and have the netting tethered by just a string within the scope, simultaneous manipulation would be greatly improved. 
Stone capture may be difficult if a stone is spherical, smooth, and greater than $2 \mathrm{~cm}$ in diameter. This leads to incomplete capture, and sliding of the nitinol frame off of the stone. In larger irregular stones, portions could be stabilized within the UN, fragmented and extracted, then repeated sequentially for the entire burden. However, in patients with significant stone burdens the indications for an open surgical approach should be individualized.

Bladder stone capture has been described using a laparoscopic entrapment sac through a 10 $\mathrm{mm}$ ports placed percutaneously in neobladders, augmented bladder, with simultaneous cystoscopic visualization (per urethra) (8). Lam et al., used a similar technique, fragmenting stones through a 30Fr sheath placed within the sac (after port removal), until small enough to remove through the $10 \mathrm{~mm}$ incision (6). Percutaneous management is becoming popular in both adults and children, and using larger sheaths improves drainage, accommodates rigid nephroscopes, and allows removal of larger stones/ fragments (3). It has also been shown to have comparable stone free rates as open surgery, in addition to being less painful and requiring shorter admission times (4).

Holmium:Yag laser cystolithopaxy is commonly performed, with advantages in being performed transurethrally or percutaneously with both rigid and flexible instruments. A downside being laser fragmentation produces smaller fragments, and a significant amount of debris (9). We have found lasers most useful in smaller stones treated transurethrally, or when anatomic challenges are present, we use flexible instrumentation. Unintentional mucosal injuries can occur when attempting to stabilize fragments against the bladder wall during fragmentation. Lasers can also be used during stone stabilization using the UN to reduce fragment dispersion, though net damage occurs with higher settings.

Designed for stone/tissue capture for extraction in the kidney or bladder, the UN's application in stabilization during lithotripsy offers several theoretical benefits. The risk of bladder injury may be lessened as the entrapped stone is maintained between the SL probe and bladder wall. In native bladders these injuries are of minimal significance, however in bowel augments or conduits they can easily cause transmural perforations. Sequestration of fragments until the pouch can be withdrawn through the 30Fr working sheath reduces the time spent in the localization and removal of loose fragments. Keeping millimeter sized particles and 'dust' trapped within the basket reduced the amount of residual stone matter that may not clear with outlet obstruction.

These theoretical benefits however, do not outweigh the limitations of this device when used for stabilization. Difficulties in simultaneous manipulation, and durability preclude it from everyday use with concomitant SL lithotripsy. The optimal size of the stone to be captured is also relatively small, though currently not defined. Though no freed netting fragments were encountered, the risk remains in foreign bodies leading to a future nidus for stones. Though alternatives may be utilized (baskets, graspers, etc), the circumferential netting provides and ideal configuration for fragmentation, without fragment spillage. Improvements in netting durability, shaft flexibility/removability and maximal diameter of applicable stone would make for an ideal instrument. Employing nitinol baskets, though resistant to fracture by SL fragmentation, would result in a similar distribution of fragments seen in non-stabilized procedures.

Limitations of this study include in vitro modeling and short trial times. Compared to typical bladder stone procedures, our trials were relatively short. However, within this time, fragmentation was significantly improved by stabilization, even with the shortcomings of the UN. The use of a 30Fr sheath with $1 \mathrm{~cm}$ stone, clinically does not require fragmentation, however as the purpose of this study was to measure fragmentation potential, extraction dynamics were not specifically investigated. Our model also may amplify shortcomings in durability as Bego stones are very hard, and by using a rigid bladder model, damage caused by the SL may have been higher than in situ. As such, an obvious limitation to the routine use of this instrument is the potential need for multiple devices.

The concept of stabilization may be useful in many situations, but with the limitations in stone size, durability and simultaneous maneuverability, the UN requires significant modifications prior to routine use. 


\section{CONCLUSIONS}

In conclusion, bladder stone stabilization during SL lithotripsy improves efficacy, however, in its current configuration the UroNet Retriever is better suited for post-lithotripsy fragment removal.

\section{CONFLICT OF INTEREST}

None declared.

\section{REFERENCES}

1. Childs MA, Mynderse LA, Rangel LJ, Wilson TM, Lingeman JE, Krambeck AE. Pathogenesis of Bladder Calculi in the Presence of Urinary Stasis. J Urol. 2012; 15: 1374-51

2. Tan YK, Weinberg AC, Kotwal S, Gupta M. Minimally Invasive Percutaneous Management of Large Bladder Stones with an Entrapment Bag Reduces Complications and Improves Operative Times. J Endourol. 2013.

3. Loeb S, Semins MJ, Matlaga BR. Novel technique for fragment removal after percutaneous management of largevolume neobladder calculi. Urology. 2012;80:474-6.

4. Docimo SG, Orth CR, Schulam PG. Percutaneous cystolithotomy after augmentation cystoplasty: comparison with open procedures. Tech Urol. 1998;4:43-5.
5. Cain MP, Casale AJ, Kaefer M, Yerkes E, Rink RC. Percutaneous cystolithotomy in the pediatric augmented bladder. J Urol. 2002;168:1881-2.

6. Lam PN, Te CC, Wong C, Kropp BP. Percutaneous cystolithotomy of large urinary-diversion calculi using a combination of laparoscopic and endourologic techniques. J Endourol. 2007;21:155-7.

7. Breda A, Mossanen M, Leppert J, Harper J, Schulam PG, Churchill B. Percutaneous cystolithotomy for calculi in reconstructed bladders: initial UCLA experience. J Urol. 2010;183:1989-93.

8. Miller DC, Park JM. Percutaneous cystolithotomy using a laparoscopic entrapment sac. Urology. 2003;62:333-6; discussion 336

9. Teichman JM, Vassar GJ, Bishoff JT, Bellman GC. Holmium:YAG lithotripsy yields smaller fragments than lithoclast, pulsed dye laser or electrohydraulic lithotripsy. J Urol. 1998;159:17-23.

Correspondence address: Shubha De, MD Glickman Urologic and Kidney Institute, Cleveland Clinic Foundation, Cleveland, Ohio, USA 2049 East 100th Cleveland, OH 44195, USA Fax: 216-445-2267 E-mail: Shubhade@gmail.com 